\title{
Perspectives on Manufacturing Engineering Software Integration
}

\author{
C. R. McLean \\ Group Leader, Manufacturing Systems Engineering \\ National Institute of Standards and Technology (NIST) \\ Gaithersburg, MD 20899 USA \\ (301) 975-3511, FAX (301) 258-9749 \\ Email: mclean@cme.nist.gov
}

\begin{abstract}
The world wide demand for integrated CAD/CAM/CAE software solutions is growing rapidly. The computer professional services business which includes systems integrators is expected to grow to meet that demand because data exchange formats and interface protocols between CAD/CAM/CAE software applications are for the most part incompatible. This incompatibility results in increased business opportunities for professional integrators who create custom integration solutions to support data exchange between software applications. These solutions are expensive to implement, require a great deal of time to develop, are very inflexible, and are not based upon industry standards. These custom solutions result in numerous problems that can be broken down into three major technical impediments: 1) the lack of understanding of what information and knowledge is shared between manufacturing design, planning, and production areas, 2) the lack of information standards that define structure and content of data that must be shared by multiple manufacturing applications, and 3) the lack of standard interface protocols between support systems, e.g., communications and database management systems, that would facilitate the sharing of information between independently-developed software applications

This paper presents perspectives on engineering tool integration issues. It also describes work underway to address those issues at NIST in Computer-Aided Manufacturing Engineering (CAME) and Systems Integration for Manufacturing Applications (SIMA) programs. Some of the technical activities include integration of design, process planning, plant layout, scheduling, and production simulation systems. A virtual production facility has been established using simulation and virtual reality systems that will provide a basis for validating manufacturing data before it is released to the shop floor.
\end{abstract}

\section{Keywords}

Manufacturing software integration, manufacturing engineering, simulation, virtual manufacturing, process modeling 


\section{INTRODUCTION}

Just as computer-aided design and engineering tools have revolutionized product design during the past decade, computer-based tools for production system engineering could revolutionize manufacturing. The major problem today is the lack of software integration--engineers need to move data between tools in a common computing environment. A recent NIST study of engineering tools has identified more than 400 engineering software products marketed today, most all of which are virtually incompatible with one another. That is, interoperability between these tools is for the most part, non-existent.

Tool kit environments are needed which integrate clusters of functions that manufacturing engineers need in order to perform related sets of tasks. Some examples of different types of tool kit environments which are needed by manufacturing engineers include:

- Manufacturing Engineering Tool Kit - Used to develop process plans for machined parts, identify manufacturing resource requirements from product design data, and validate those plans using simulation technology.

- Production System Engineering Tool Kit - Used to translate product design, production demand forecasts, and other constraints into a production system design. Also provides tools for evaluating the performance of candidate designs.

- Business Process Re-engineering and Producibility Analysis Tool Kit - Used to analyze and re-design business processes associated with manufacturing and evaluate the product producibility, i.e., the relative ease by which a process may be produced.

- Quality Engineering Tool Kit - Used to develop quality models and metrics for processes, gather and evaluate statistical process control data, and initiating fine tuning of process parameters.

If these tool kit environments were available today, they would not only be used by manufacturing engineers, but also by product designers, quality engineers, industrial engineers, system engineers, process planners, tool designers, and manufacturing management. In the future, new users may include: manufacturing strategists, producibility engineers, enterprise designers, benchmarking teams, etc.

\subsection{NIST Programs in Manufacturing Software Integration}

The NIST Systems Integration for Manufacturing Applications (SIMA) Program, Barkmeyer (1995) and the NIST/Navy Computer-Aided Manufacturing Engineering (CAME) Program, McLean (1993) are focusing on providing the models, frameworks, operating environment, common databases, and interface standards for integrating a wide variety of tools for designing manufacturing processes, equipment, and enterprises.

In collaboration with industry, the CAME program is assessing needs with respect to manufacturing engineering tool integration. The program is developing architectures, interfaces, and databases for integrating engineering tools environments. Prototype integrated engineering tool kits are also being constructed using commercial products to validate interface specifications. Integrated tool kit solutions will be evaluated at industry sites. The principal elements of the program's technical approach are:

1) Identify and address critical industrial needs through collaboration,

2) Develop solutions to engineering tool integration problems, 
3) Construct prototype environments using commercial products,

4) Validate results through industrial testing of system implementations,

5) Specify and promote needed industry standards, and

6) Facilitate the rapid commercialization of new technology.

A study conducted by the U.S. Department of Defense suggested that industry can obtain major economic benefits from increased investment in: 1) integration methodologies, 2) simulation and modeling, and 3) manufacturing/industrial engineering support tools. Some of the benefits obtained from the CAME program are outlined below.

\section{Improved engineering function}

Engineering tool kit technology will help manufacturing engineers make better decisions and more quickly evaluate the effects of those decisions. By improving process planning and simulation capabilities, a much greater percentage of products will be produced correctly the first time. Furthermore, the overall time to perform the engineering function will also be reduced if fewer changes to plans and programs are required once a job hits the shop floor. These improvements will result in fewer scrapped parts and less re-work. The integration of software packages and common databases will ensure that less time is wasted re-entering the same data into multiple engineering tools.

\section{General productivity benefits}

A number of broader benefits will be seen as a result of improvements in the manufacturing engineering function. There will be better utilization of shop floor equipment. Shops will be able to respond quicker to rush orders if their resources are used more efficiently. More energy can be devoted to producing higher quality products. Shorter response times will be realized for spare/repair parts for existing products as well as new products.

\section{Increased availability of engineering products}

Finally, the cost of performing the engineering function will be reduced if commercial software and hardware can be made more accessible to a larger group of users. This project will help increase the interoperability and value added by engineering tools. It is likely that this will lead to a greater demand and market for these commercial products. As the sales of these products increase, the "per unit" costs of acquiring software at new sites should be expected to go down.

\subsection{Overview of Paper}

The remainder of this paper describes the work underway at NIST using one of several engineering tool integration projects as an example of our technical approach. The project is focusing on developing interfaces for integrating tools for production system engineering. Section 2 of the paper provides an overview of the top two levels of the process model for production system engineering which has been developed to guide tool integration. Section 3 describes the integrated manufacturing engineering tools environment, the virtual manufacturing system which is being used to test tool integration today, and outlines future work.

\section{PRODUCTION SYSTEM ENGINEERING PROCESS MODEL}

The Production System Engineering Tool Kit under development by NIST and collaborators will provide functions to specify, design, engineer, simulate, analyze, and evaluate a production system. 
Other functions included within the environment are project management and budgeting. Examples of production systems which may eventually be engineered using this environment include: transfer lines, group technology cells, automated or manually-operated workstations, customized multipurpose equipment, and entire plants. The initial focus for this project is on small production lines used to assemble power tools. A process model is being used to define interfaces which are needed to integrate the tool kit.

A process model is one of several models that are needed to implement an integrated engineering tools environment. The process model defines the functions that tools must perform in order to engineer a production system. The model also defines inputs, outputs, controls, and mechanisms for carrying out the functions. The process model is a key reference document for defining the data flows and interfaces between the modules in the integrated environment.

The process model for production system engineering has been developed using Integrated Definition Method $\left(\mathrm{IDEF}_{0}\right)$ modeling techniques and the Meta Software Design/IDEF TM tool, see Meta (1994). The model defines the tool kit functions and data inputs/outputs for each function. Detailed information models are under development which further specify each data input and output identified in the process model. The information models are being used to implement shared databases, exchange files, messages, and program calls for passing information between the commercial software tools.

The zero level of the model identifies the production system engineering function, its inputs, and its outputs. The first level of the model decomposes the engineering process into five major functions or activities: 1) define the production system engineering problem, 2) specify production processes required to produce the product, 3) design the production system, 4) model the system using simulation and evaluate its performance under expected operating conditions, and 5) prepare plans and budgets. Inputs to the production system engineering function include: production requirements, product specifications, quality, time, and cost constraints, and manufacturing resources. Outputs of the function include: process specifications, simulation models, performance analyses, system specifications, implementation plans, and budgets.

Figures 1 and 2 illustrate the first two levels of the IDEF0 model. The model further decomposes each of these functions and data flows into sub-levels. Brief summaries of the sublevels are presented in the sections that follow.

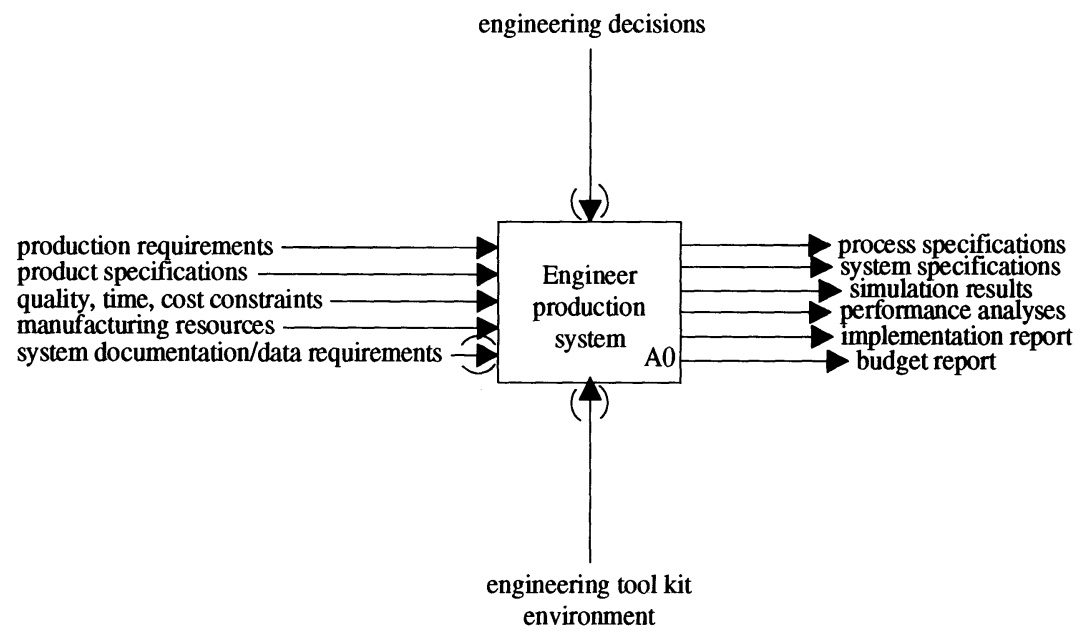

Figure 1 Top level of the production system engineering IDEF model. 


\subsection{Engineering Problem Definition}

The first step in engineering the production system is clearly identifying the production engineering problem which is to be solved. Problem definition data will influence how all of the other production engineering functions are carried out. This activity is primarily one of gathering and organizing data from a number of different sources. Ultimately, data gathered as a part of this activity would be recorded in template forms, imported from other applications, and maintained in a shared database. Critical data which must be identified to initiate the engineering process includes:

- Product data and key product attributes - product name, part number, model number, description, functionality, product structure (bill of materials), material composition, dimensions, weight, reference drawings, part geometry models, part family or group technology classification codes, technical specifications, reference documents,

- Production system and engineering project type - new production system (e.g., plant, line, cell), modification to existing system (i.e., product or process changes), relocation of system to new site, phasing out of a production system,

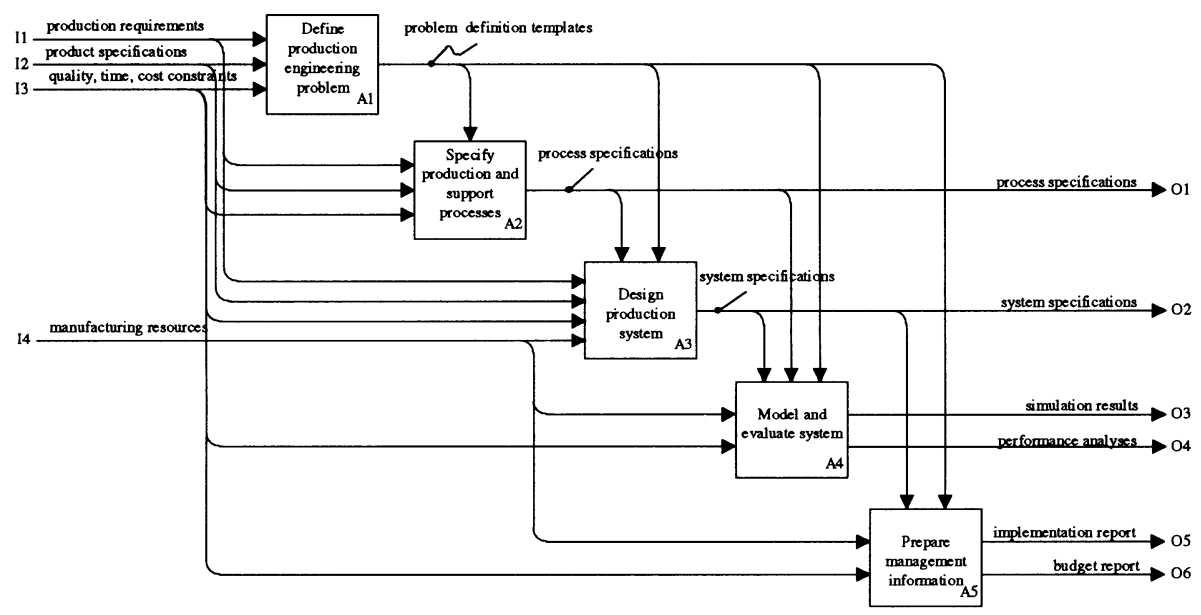

Figure 2 First level of decomposition of the production system engineering model.

- Manufacturing constraints and issues - market forecast and production rates required (minimum, normal and peak production rates in units/hour, units/shift, units/day, units/year), production capacity, level of automation versus manual operation expected, information and control system requirements, target production site(s), floor space limitations, quality and yield requirements, safety stock requirements, storage availability, known environmental or safety hazards, production plant calendar,

- Critical milestone dates and schedules - production ramp up plan, target dates for: system requirements specified, system design completed, requests-for-proposals issued, systems installed, testing completed, training completed, system operational, post production support, system phase out,

- Expected or estimated costs - product price, manufacturing cost, system implementation, operating costs, 
- Manufacturing data for related products - production engineering data for this or previously manufactured products (in some cases all outputs from previous engineering projects), competitor products and sites, possible benchmarking sites.

With the exception of critical milestone dates, most of the information outlined above may at some point be used by the next function in the process model, i.e., the specification of production and support processes. All data may be used directly by other downstream functions, if appropriate. During the course of the production system engineering process, downstream functions may provide feedback suggesting changes to the problem definition data.

\subsection{Production And Support Process Specification}

The second phase of the production system engineering activity is to develop a process specification for the production and support operations required to manufacture the product, see Tanner (1985), Salvendy (1992), and Sule (1994). Data developed during this phase will ultimately take the form of directed graphs and/or flowcharts. Nodes in the graphs will contain attributes which identify processes and their parameters.

A manufacturing/assembly precedence structure diagram is developed from the product geometry data and bill of materials. From the precedence structure, processes and processing precedence constraints may be derived. The derivation process may be based on human experience and intelligence, or implemented as a rule-based expert system. Data developed by this function includes:

- Process identification - process name, process type (operation, storage, inspection, delay, transportation, information, or combined activity), process parameters,

- Process resources - input product components, output product (subassembly or part identifier), tooling and fixtures, staff and job skill requirements, process by-products and hazards,

- Process time and costs - process duration, estimated process cost, product value-added.

This process is recursive--high level processes are decomposed into sub-processes until all basic or primitive operations are specified. Constraints on groups of processes and operations are identified and precedence relationships are specified.

Process specifications are perhaps best represented as diagrams and/or tables. Graphical editing functions and human interaction are normally required to layout diagrams in an understandable form. Large diagrams may be unwieldy and should be decomposed into multiple levels of sub-diagrams.

Other process specification data which may be developed as part of this phase include:

- activity relationship matrices are defined which describe how different processes relate to each other, e.g., required proximity or location.

- specification of requirements for processes, tooling, job skills, timing and line balancing, quality control, process audits,

- development of process and inspection plans, process description sheets,

- development of time standards for operations,

- ergonomic analyses of manual tasks,

- value engineering analysis (i.e., determination of job activities/steps which can be eliminated).

Processing scenarios may also be defined which describe how production will be carried out before, during, and after the new production system is implemented. 
Process specifications next must be reviewed and revised to correct errors, inconsistencies, etc. Feedback requesting changes to the problem definition, as the process specification is developed. As the system design is developed in the next phase, feedback may be provided indicating required changes in process specifications.

\subsection{Production System Design}

The third phase of the engineering process is production system design. This activity includes the design of the physical processing systems, material storage and delivery systems, and information management/control systems for the production system. The production system design problem is addressed in Sule (1994). The mechanical assembly system and flexible manufacturing system problems are described, respectively in Nevins (1989) and Draper (1984). Facility layout is presented Apple (1977) and Francis (1992). Manufacturing system architecture, design, and specification development processes are defined in Rechtin (1991), Bertain (1987), Rembold (1993), Compton (1988), and Purdy (1991).

A generic decomposition of production system design is: 1) define system requirements for each process, 2) assign requirements to system modules, 3) develop system operating scenarios for the modules, 3) identify candidate systems/machines/tooling for each module, 4) evaluate alternative technologies and candidate offerings, 5) determine number of systems required based on processing cycle time and required throughput, 6) conduct system build or buy analyses, 7) select systems for acquisition, and 8) developed detailed design for overall system based upon build and buy decisions.

The generic production system design process can also be viewed in terms of the specific types of systems involved, i.e., process, logistics support, and information. The remainder of this section briefly summarizes considerations associated with the design of each of these elements of the overall production system.

The design of the processing system involves: the selection of a hierarchy of processing systems to implement the modules (including plants, centers, lines, cells, stations, equipment, devices, and tooling), assignment of processes to the systems, estimation of resource utilization levels, and balancing of production systems.

The design of the logistics systems can be divided into two related problems: production material logistics and plant logistics. Production material logistics includes: determination of production material requirements (raw materials, components, packaging, carriers), estimation of consumption rates, determination of source selection strategies (make-or-buy analyses), lead times, and shipping (air/land/sea) methods for source materials.

Plant logistics concerns the systems which move and store materials within the facility. Plant logistics involves: determination of floor space and volumetric requirements for each process/machine/system, identification of production and tooling material storage requirements (i.e., loading docks, staging areas, centralized storage areas, line side storage), selection of storage systems (i.e., automated storage and retrieval systems, manual storage systems, production line buffers and feeders), specification of material flow through the facility (i.e., raw materials, components, work-in-process, and finished goods from the dock to lines through lines and back to dock), selection of material handling systems (e.g., hand truck, fork lift, conveyor, automated guided vehicles), determination of stock replenishment strategies, design of safety and environmental systems, development of physical plant layout in two and three dimensions, and evaluation of logistics system for further production capacity growth capabilities.

Production information systems may include: monitor and control systems, communications, display and user interface systems, database management systems and their 
databases, data collection systems, production information systems, peripheral devices (e.g., printers, magnetic scanners, monitors, bar code readers, infrared tracking systems), production accounting and reporting, statistical process/quality control (SPC/SQC) systems, time and attendance recording, and preventive/corrective maintenance support systems. The information systems design activity includes: requirements specification, architecture development, process and information modeling, detailed design, interface specification, integration and test planning, and user documentation development.

The outputs of the production system design phase are detailed system specification documents. This phase may provide feedback to problem definition and process specification phases indicating changes which must occur as the result of design analyses. The next phase is the simulation modeling of the system which has been specified by production system design.

\subsection{Simulation Modeling Of The System}

Once a design, or partial design, for the production system is specified, it should be modeled and evaluated using simulation technology. The purpose of this phase is to better understand the dynamics of the proposed system and help ensure that it satisfies the constraints outlined in the problem definition phase. Inputs to this phase are derived from all of the previous phases. Pegden (1990), Askin (1993), and Carrie (1988) describe the simulation modeling process. Knepell (1993) describes the evaluation and validation of models.

The first step in developing a simulation model for the system is to define a problem statement and simulation objectives, i.e., what is expected to be learned from the simulation model. The types of alternative models to be considered and constructed need to be identified, e.g., discrete event simulation, material flow, system mechanics and kinematics, ergonomic, and/or manufacturing process. Appropriate simulation tools must be selected based on the types of models to be constructed. Next, system performance measures must be identified. Some examples of performance measures include: throughput, cycle time, work-in-process, machine downtime, and machine utilization.

Next, the system simulation model elements and their behaviors must be specified. Model elements used will depend on the types of simulations to be constructed. Elements of these models may include the attributes associated with: manufacturing resources, servers, queues and selection criteria, work pieces/loads/objects, arrival distributions, processes, system movements and material flows, timing distributions, failure and repair rates, etc. The information needed to derive the model elements will be drawn from problem definition, process specification, and system design data. The actual simulation models may then be constructed using the selected simulation tools.

Another critical activity in the modeling and evaluation phase is the development of test data for the simulation runs. This activity includes: identification of data sources, gathering of test data, formatting and loading the data, and determining the number of simulation runs required to produce significant results. Once the simulation has been constructed and the test data has been loaded, the models can be run and evaluated.

The simulations must be validated, i.e., it is necessary to determine whether results are believable based on experience, other data, etc. There are two aspects to this problem: 1) does the simulation program behave as expected, and 2) does the outcome reflect reality. If the results are not correct or creditable, either the simulation must be fixed, models modified, or the test data may need to be changed. Some examples of evaluations that may be performed on the results include: verification of the accuracy of model, analysis of errors and failures, bottlenecks, throughput, flow time, expected yields and quality, interference problems, collisions, etc. 
After the results of the simulation are reviewed, it may be necessary to revise design specifications and the system models, process specifications, or even basic assumptions spelled out in the problem definition. Some of the results of simulation, e.g., timing data, may be fed forward in to the engineering project management phase.

\subsection{Engineering Project Management}

Another parallel phase in production system engineering is the development of engineering project management data. Project management and budgeting is described in Kerzner (1984). These functions include: development of project plans, preparation of budgets, establishment of configuration management controls, and generation of reports. Principal inputs to this activity include: problem definition and system design specification data. Timing information may be drawn from simulation results.

Project planning involves the definition of the production system engineering project in terms of phases, tasks, resources, and timing data. Possible phases may include: feasibility study, planning, needs and requirements analysis, detailed design, acquisition and installation, testing, training, pilot and full production operation, and phase out. Critical milestones are identified as part of the phase definition activity.

Each major project phase is specified in terms of tasks and sub-tasks. Task precedence constraints and overlap options are identified. Required resources associated with each task are identified. Staff responsibilities are specified on each task. Resource balancing may be required. Timing information is also estimated for each task, including: expected or required start, end dates, estimated task durations and lead times. From this data, schedules may be generated and critical paths determined.

Cost factors and their analysis is an extremely important part of the system design and implementation process. Malstrom (1984) provides detailed guidance on the development of manufacturing cost estimates and budgets. Budget cost categories that may be considered include: project phase, planning, labor, tooling, capital equipment, projected maintenance, information and control system, operational, training, licensing and inspection, construction, installation, material (components, consumables), overhead (utilities, labor multipliers, area usage), and rental costs.

The budgeting process includes: gathering of cost data, entering data into spreadsheets or databases by budget categories, projecting estimates where data is unavailable, generating summaries by categories, and producing budget reports. Budgeting data is reviewed for significant deviations from targets and opportunities for savings are identified. Budget data is then used to generate feedback, if required, to the problem definition and production system design phases.

Another critical activity included in this phase is the configuration management of engineering data and project documents. Principles of configuration management are outlined in Daniels (1985). This activity includes: identification of key documents, definition of revision control-review-promotion policies and procedures, identification of organizational responsibilities, establishment of notification procedures for project staff, establishment of security policies and access control mechanisms, and the placement of documents and data under configuration management.

The final activity in the management area is generation and publication of reports that summarize the results of each of the other phases. Functions included in this activity include: outline development, document editing and assembly, layout and formatting, and printing. This activity draws input from all of the other functions in this phase and the other phase. 
Once plans, budgets, configuration management policies, and reports are completed they need to be reviewed to ensure that they are realistic and meet the requirements established in the problem definition phase. If not, either the plans need to be changed or information must be fed back to problem definition and/or system design to re-scope the system.

\section{INTEGRATED ENGINEERING TOOLS ENVIRONMENT}

The process model for production system engineering is being implemented as an integrated tools environment through the collaborative efforts of NIST, academia, and industry. Black and Decker Corporation is collaborating on the production system engineering process and providing test data on production lines. Although a number of engineering tool vendors have provided software for integration into the environment, the final selections of all required software tools has not been completed.

The production engineering environment is being implemented on a high performance personal computer and Silicon Graphics ${ }^{\mathrm{TM}}$ engineering workstation. Commercial software tools used in the implementation of the engineering environment include: a business process reengineering - flowcharting package, a plant layout system, a computer-aided design system, a manufacturing simulation system, a spreadsheet tool, a project management system, and a relational database management system. Other tools are under consideration for incorporation into the environment at a future time.

A virtual manufacturing system has been implemented using the simulation system to support the tool kit project. The virtual manufacturing facility currently provides simulation models to aid the engineer in the validation of engineering data. The virtual manufacturing facility currently consists of the following manufacturing areas (see Figure 3): tool room, shipping, receiving, heat treat, paint, manufacturing engineering administrative offices, and three machining areas.

The interoperability of the commercial engineering tools that are available today is extremely limited. As such, users must re-enter data as they move back and forth between different tools carrying out the engineering process. Project collaborators are working with NIST to address this problem. Collaborators will: define generic information models for production system engineering data, specify interfaces for integrating tools, develop prototype integrated environments and shared databases, and implement test case production system engineering projects. Examples of the types of shared data under consideration by the collaborators for the common database includes: production requirements, product specifications, process specifications (diagrams, flowcharts, plans, routings, operation sheets, programs), equipment specifications, budget spreadsheets, project plans, simulation models and model elements, setup illustrations, plant layouts, information models, interface specifications, system descriptions, estimated yield data, process capabilities, and quality data.

A long term objective of the project is to improve the productivity of users by creating an integrated environment where changes to data and decisions automatically percolate through the various tools contained within system. Project results will provide a basis for defining interface standards that will facilitate the integration and interoperability of commercial tools in the future. 


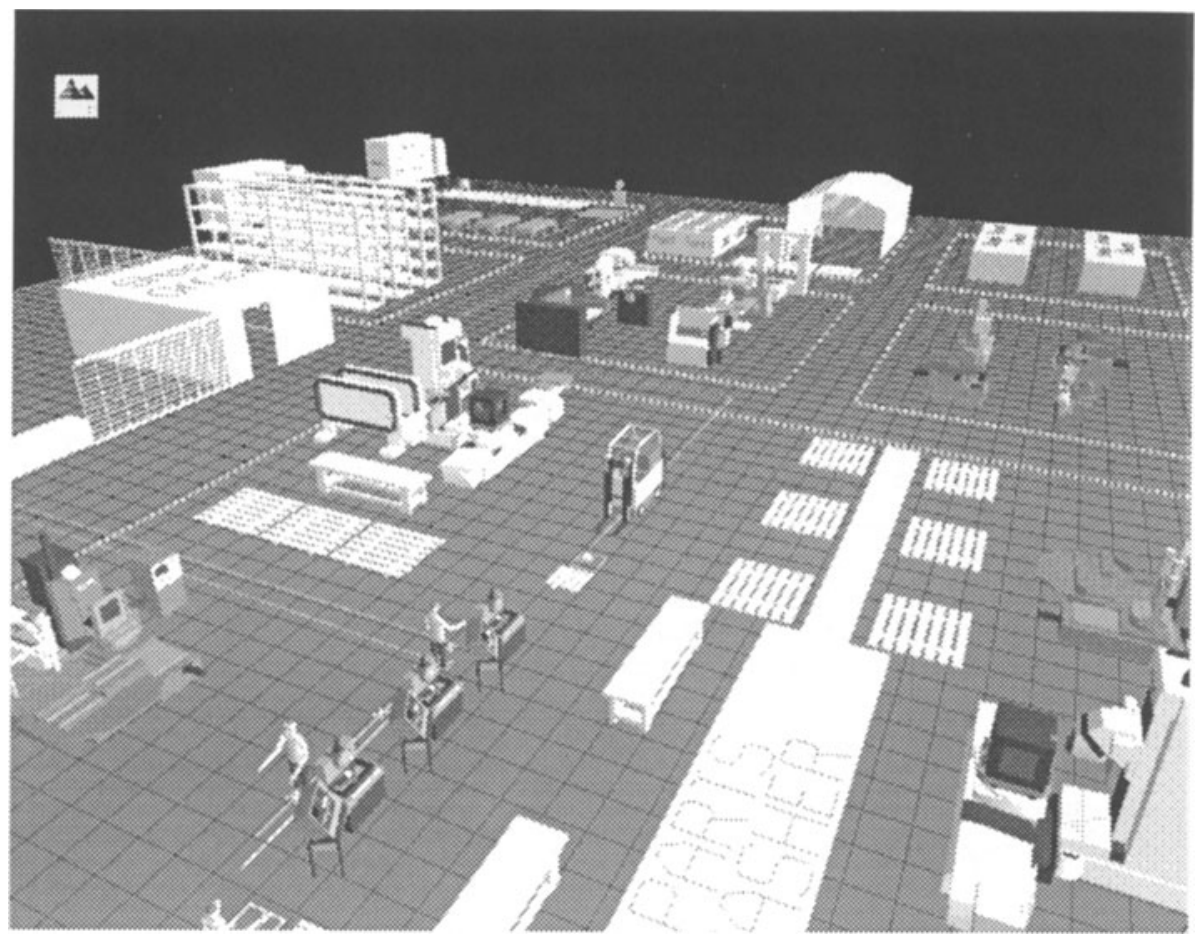

Figure 3 Virtual manufacturing system implemented at the National Institute of Standards and Technology.

The author wishes to acknowledge contributions to this paper from Mike Iuliano, Swee Leong, and Dr. Albert Jones. Work described in this paper was sponsored by the U.S. Navy Manufacturing Technology Program and the NIST Systems Integration for Manufacturing Applications (SIMA) Program. No approval or endorsement of any commercial product by the National Institute of Standards and Technology is intended or implied. The work described was funded by the United States Government and is not subject to copyright.

\section{REFERENCES}

Apple, J.M., (1977) Plant Layout and Material Handling, John Wiley and Sons, New York, NY.

Askin, R.G., Standridge, C.R. (1993) Modeling and Analysis of Manufacturing Systems, John Wiley and Sons, New York, NY.

Barkmeyer, E.J., Hopp, T.H., Pratt, M.J., Rinaudot, G.R., editors (1995) Background Study: Requisite Elements, Rationale, and Technology Overview for the Systems Integration for Manufacturing Applications Program, NIST Technical Report, Gaithersburg, MD.

Bertain, L., Hales, L. (1987) A Program Guide for CIM Implementation, Society of Manufacturing Engineers, Dearborn, MI.

Carrie, A. (1988) Simulation of Manufacturing Systems, John Wiley and Sons, Chichester, Great Britain. 
Compton, W.D., editor (1988) Design and Analysis of Integrated Manufacturing Systems, National Academy Press, Washington, DC.

Daniels, M.A. (1985) Principles of Configuration Management, Advanced Applications Consultants, Rockville, MD.

Draper Laboratory Staff (1984) Flexible Manufacturing Systems Handbook, Noyes Publications, Park Ridge, NJ.

Francis, R.L., McGinnis, Jr., L.F., White, J.A. (1992) Facility Layout and Location: An Analytical Approach, Prentice-Hall, Englewood Cliffs, NJ.

Kerzner, H. (1984) Project Management: A Systems Approach to Planning, Scheduling, and Controlling, Van Nostrand Rheinhold, New York, NY.

Knepell, P.L. and Arangno,D.C. (1993) Simulation Validation: A Confidence Assessment Methodology, IEEE Computer Society Press.

Malstrom, E.M. (1984) Manufacturing Cost Engineering Handbook, Marcel Dekker, NY.

McLean, C.R. (1993) "Computer-Aided Manufacturing Systems Engineering" in IFIP Transactions B-13 Advances in Production Management Systems, North-Holland, Amsterdam, Netherlands.

Meta Software Corp. (1994) Design/IDEF User's Manual and Tutorial For Microsoft Windows, Meta Software Corp., Cambridge, MA.

Nevins, J.L., Whitney, D.E., (1989) Concurrent Design of Products and Processes: A Strategy for the Next Generation in Manufacturing, McGraw-Hill, New York, NY.

Pegden, C.D., Shannon,R.E., Sadowski,R.P. (1990) Introduction to Simulation Using SIMAN, McGraw-Hill, New York.

Purdy, D.C. (1991) A Guide to Writing Successful Engineering Specifications, McGraw-Hill, New York, NY.

Rechtin, E., (1991) Systems Architecting: Creating and Building Complex Systems, PrenticeHall, Englewood Cliffs, NJ.

Rembold,U., Nnaji, B.O., Storr, A. (1993) Computer Integrated Manufacturing and Engineering, Addison-Wesley, Wokingham, England.

Salvendy, G., editor (1992) Handbook of Industrial Engineering, John Wiley and Sons, New York, NY.

Sule, D.R., (1994) Manufacturing Facilities: Location, Planning, and Design, PWS Publishing Company, Boston, MA.

Tanner, J.P. (1985) Manufacturing Engineering: An Introduction to Basic Functions, Marcel Dekker, New York, NY.

\section{BIOGRAPHY}

Charles McLean is Leader of Manufacturing Systems Engineering Group in the Manufacturing Systems Integration Division at the U.S. National Institute of Standards and Technology. He has managed research programs in manufacturing automation at NIST since 1982. He has served as System Architect for the Automated Manufacturing Research Facility (AMRF) and Program Manager for the National PDES Testbed. His group is conducting research in engineering tool integration, manufacturing data validation, production systems engineering, manufacturing simulation, and production scheduling. He holds a Master of Science in Information Engineering from University of Illinois, Chicago, IL, and a Bachelor of Arts degree from Cornell University, Ithaca, NY. 